\title{
An Intrinsically Disordered Pathological Variant of the Prion Protein Y145Stop Transforms into Self-Templating Amyloids via Liquid-Liquid Phase Separation
}

Aishwarya Agarwal, ${ }^{\dagger}$ Sandeep K. Rai ${ }^{\dagger}$ Anamika Avni, and Samrat Mukhopadhyay*

Centre for Protein Science, Design and Engineering, Department of Biological Sciences, and Department of Chemical Sciences, Indian Institute of Science Education and Research (IISER), Mohali, Knowledge City, Mohali, Punjab, India

${ }^{\dagger}$ Contributed equally

*Corresponding author. Email: mukhopadhyay@iisermohali.ac.in 


\begin{abstract}
Biomolecular condensation via liquid-liquid phase separation of intrinsically disordered proteins/regions (IDPs/IDRs) along with other biomolecules is thought to govern critical cellular functions, whereas, aberrant phase transitions are associated with a range of deadly neurodegenerative diseases. Here we show, a naturally occurring pathological truncation variant of the prion protein $(\mathrm{PrP})$ by a mutation of a tyrosine residue at 145 to a stop codon (Y145Stop) yielding a highly disordered N-terminal IDR that spontaneously phase-separates into liquid-like droplets. Phase separation of this N-terminal segment that is rich in positively charged and aromatic residues is promoted by the electrostatic screening and a multitude of other transient, intermolecular, noncovalent interactions. Single-droplet Raman measurements in conjunction with an array of bioinformatic, spectroscopic, microscopic, and mutagenesis studies revealed that the intrinsic disorder and dynamics are retained in the liquid-like condensates. Lower concentrations of RNA promote the phase transition of Y145Stop at low micromolar protein concentrations under physiological condition. Whereas, higher RNA to protein ratios inhibit condensation indicating the role of RNA in modulating the phase behavior of Y145Stop. Highly dynamic liquid-like droplets eventually transform into dynamically-arrested, ordered, $\beta$-rich, amyloid-like aggregates via liquid-to-solid transition upon aging. These amyloid-like aggregates formed via phase separation display the self-templating characteristic and are capable of recruiting and autocatalytically converting monomeric Y145Stop into amyloid fibrils. In contrast to this disease-associated intrinsically disordered Y145 truncated variant, the wild-type full-length PrP exhibited a much lower propensity for phase separation and liquid-to-solid maturation into amyloid-like aggregates hinting at a potentially crucial, chaperone-like, protecting role of the globular C-terminal domain that remains largely conserved in vertebrate evolution. Such an intriguing interplay in the modulation of the protein phase behavior will have much broader implications in cell physiology and disease.
\end{abstract}




\section{Introduction}

A growing body of current research has revealed that in addition to canonical membraneenclosed organelles, eukaryotic cells also contain organelles without delimiting membranes. These membrane-less organelles, also known as biomolecular condensates, are formed via liquid-liquid phase separation (LLPS) of proteins, nucleic acids, and other biomolecules. ${ }^{1-16}$ These mesoscopic intracellular compartments are dynamic, liquid-like, permeable, nonstoichiometric supramolecular assemblies that allow rapid exchange of components within the environment and offer unique spatiotemporal control of the macromolecular organization and cellular biochemistry. Intracellular phase transitions are governed by an intricate balance between the enthalpy and entropy of mixing and are primarily driven by intrinsically disordered proteins/region (IDPs/IDRs) containing prion-like low-complexity regions (LCRs) comprising a "stickers-and-spacers" sequence architecture. 1,17-28 The sticker residues can promote weak, stereospecific, multivalent, transient intermolecular contacts involving electrostatic, hydrophobic, hydrogen bonding, dipole-dipole, $\pi-\pi$, and cation- $\pi$ interactions. Whereas, the flexible spacer residues can dynamically control the making-and-breaking of noncovalent interactions giving rise to a liquid-like behavior of phase-separated biomolecular condensates. While liquid-like intracellular bodies are lined with a diverse array of cellular functions, liquid-to-gel and liquid-to-solid phase transitions are implicated in a range of debilitating neurodegenerative diseases. ${ }^{29-39}$ Therefore, there is an emerging consensus on the central role of biomolecular condensation and maturation promoting more persistent intermolecular contacts in aberrant pathological phase transitions.

The conformational conversion of the human prion protein $(\mathrm{PrP})$ into a misfolded, $\beta$ rich, aggregated, self-replicating amyloid-like form is associated with a range of invariably fatal and transmissible neurodegenerative diseases classified under transmissible spongiform encephalopathies. ${ }^{40,41}$ This class of diseases is either caused by a spontaneous prion misfolding leading to sporadic Creutzfeldt-Jakob disease (CJD) or due to mutations in the prnp gene resulting in familial CJD, fatal familial insomnia, and Gerstmann-SträusslerScheinker (GSS) syndrome. ${ }^{40-42}$ Cellular PrP is 253 -amino acids protein consisting of an Nterminal signal peptide (residues 1-23) that is cleaved upon maturation, a positively charged unstructured N-terminal tail (amino acids 23-120), a globular C-terminal domain (amino acids 121-231), and a GPI-anchor signal (residues 231-253) (Figure 1A,B). ${ }^{40-43}$ The Nterminal unstructured domain can be classified as an IDR that comprises two lysine clusters (residues 23-30 and 100-110), five glycine-rich octapeptide repeats (PHGGGWGQ), and a 
hydrophobic segment (113-135). In contrast, the C-terminal domain is highly structured and consists of three $\alpha$-helices (amino acids 144-154, 175-193, and 200-219) and two short antiparallel $\beta$-strands (residues 128-131 and 161-164) (Figure 1B). In vitro prion conversions and amplifications from recombinantly expressed PrP recapitulate several key structural and biochemical characteristics of pathogenic prion deposits. ${ }^{44-46}$ An unusual pathological mutation of the tyrosine at residue 145 to a stop codon (PrP-Y145Stop) results in a Cterminally truncated intrinsically disordered variant of the protein that exhibits GSS-like phenotype. ${ }^{47-49}$ Under normal conditions, this fragment is unstable and is rapidly degraded in the cell, however, under stress conditions, Y145Stop accumulates in the endoplasmic reticulum, Golgi, and nucleus. This truncation mutation results in pathological amyloid deposits in the brain. ${ }^{50,51}$ In this work, we show that intrinsically disordered Y145Stop spontaneously phase-separates into highly dynamic liquid-like droplets that undergo a timedependent maturation into ordered, solid-like, self-templating amyloid aggregates via a liquid-to-solid phase transition. The prion phase behavior can be modulated by RNA that tunes the material property of these condensates. The LLPS-mediated conformational switch of Y145Stop provides a mechanistic underpinning of effective nucleation and transition into self-replicating pernicious amyloid-like species.

\section{Results}

\section{PrP Y145Stop undergoes liquid-liquid phase-separation in vitro}

In order to examine the presence of intrinsic disorder and LCRs in Y145Stop, we first used well-known bioinformatic prediction tools ${ }^{52-56}$ which together revealed the presence of two LCRs (residues 50-94 and 113-135) and the highly disordered nature of Y145Stop. (Figure 1B-F). This was further verified experimentally by monitoring circular dichroism (CD) of recombinantly expressed human prion protein Y145Stop (Figure 1G). Next, we used phase separation prediction tools such as FuzPred ${ }^{54}$ and catGRANULE ${ }^{55}$ that revealed a high LLPS propensity of intrinsically disordered Y145Stop (Figure 1E,F). To experimentally verify the LLPS propensity of PrP Y145Stop, we began by characterizing its phase behavior in vitro using turbidity measurements and confocal microscopy. Under cytosolic $\mathrm{pH}(\mathrm{pH} 7.5)$ at 37 ${ }^{\circ} \mathrm{C}$ Y145Stop even at a high protein concentration $(100 \mu \mathrm{M})$ remained clear and dispersed. Upon addition of increasing amounts of salt, the turbidity of the protein solution rose immediately indicating phase separation under cytosolic conditions (Figure $1 \mathrm{H}$ ). We next created a single-Cys mutant at residue position 31 of Y145Stop in order to label the protein 


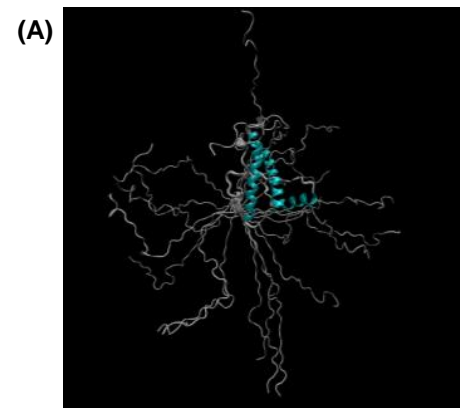

(C)

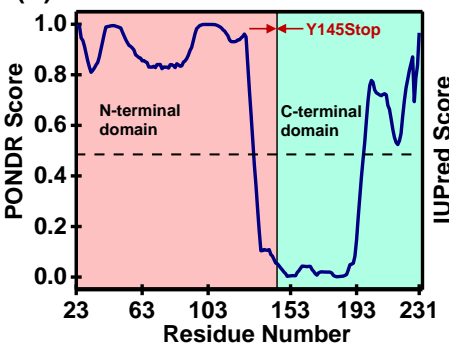

(G)

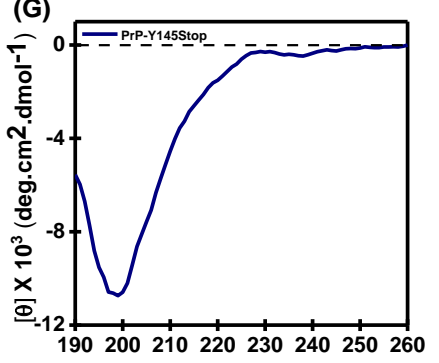

(L)

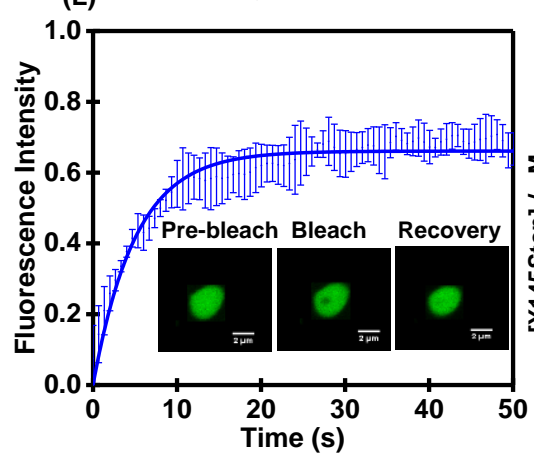

23
(B) Octapeptiderepeats

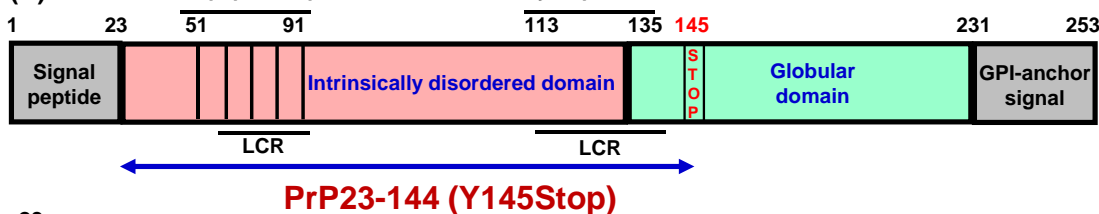

KKRPKPGGWN TGGSRYPGOG SPGGNRYPPO GGGGWGOPHG GGWGOPHGGG WGQPHGGGWG QPHGGGWGQG GGTHSQWNKP SKPKTNMKHM AGAAAAGAVV GGLGGYMLGS AMSRPIIHFG SD ${ }^{144}$
(D)

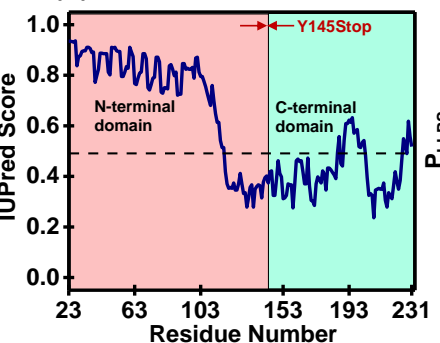

(I)
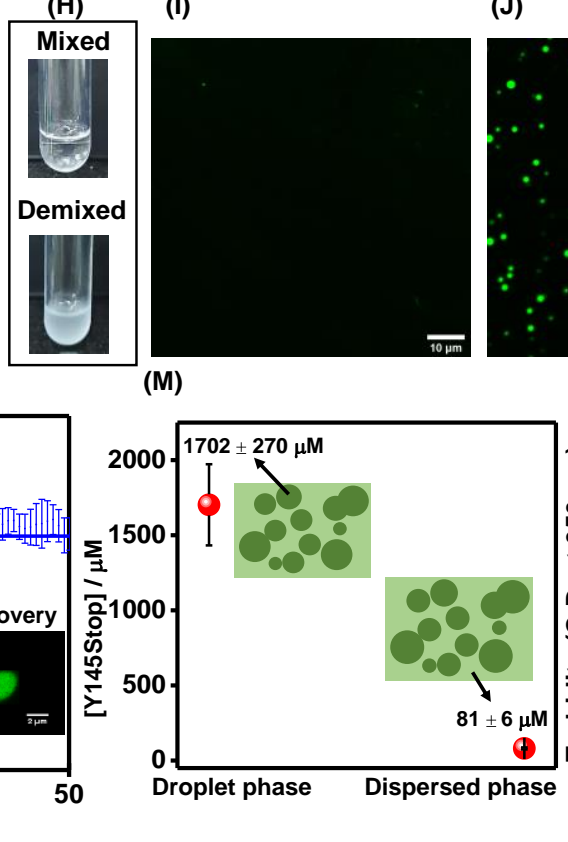

(E)

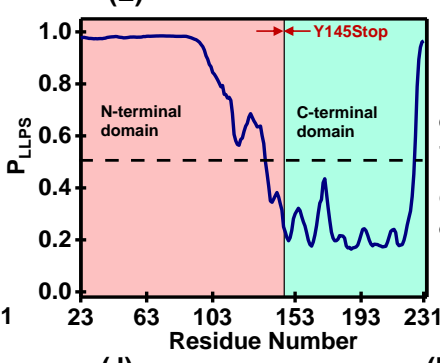

(J)

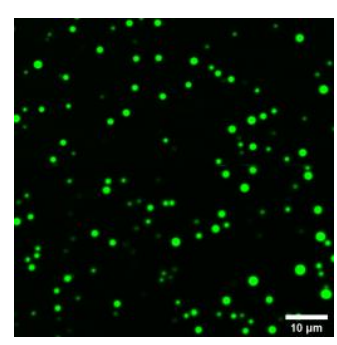

(N)

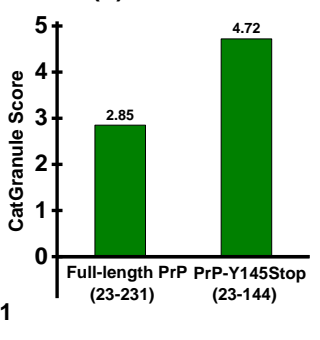

(K)

Droplet fusion Surface-wetting
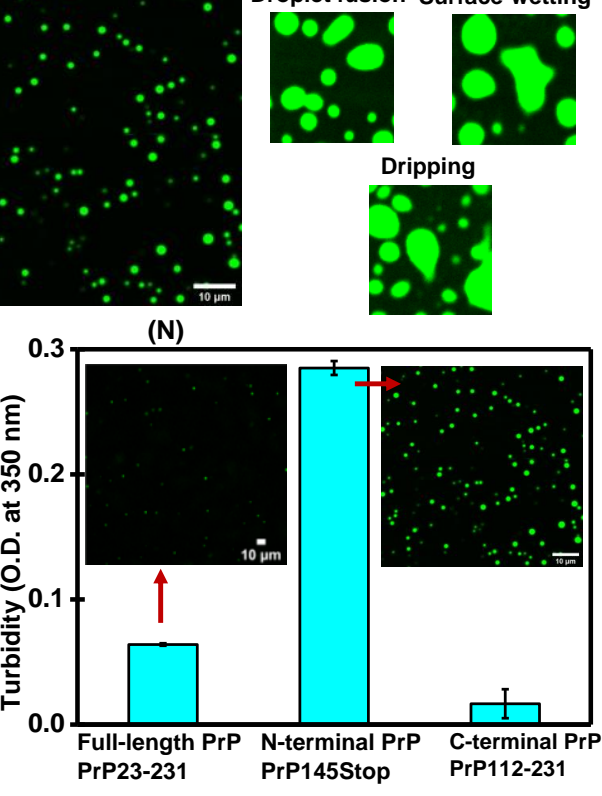

Figure 1. Phase separation of PrP-145Stop. (A) Overlay of 20 conformations obtained from the NMR structure of human PrP90-231 (PDB ID: 2LSB) ${ }^{43}$ generated using PyMOL (Schrödinger, LLC, New York) showing conformational heterogeneity at the N-terminal domain. (B) Schematic representation of PrP indicating all the segments and domains (Y145Stop is also highlighted). The amino acid sequence of Y145Stop from residues 23 to 144 showing positively charged residues (blue) and aromatic residues (green). Two low complexity regions (LCRs) predicted by SMART (Simple Modular Architecture Research Tool $)^{56}$ are underlined. The disorder predictions tools PONDR $^{52}$ (C) and IUPred ${ }^{53}$ indicate highly unstructured N-terminal segment of PrP. A boundary between disordered and ordered regions is shown at residues 145 (this boundary is conventionally shown at residue 
121). Prediction of LLPS propensity using FuzPred ${ }^{54}(\mathrm{E})$ and catGRANULE ${ }^{55}$ (F). The LLPS probability ( $\mathrm{P}_{\text {LLPS }}$ ) of Y145Stop is 0.997 obtained from FuzPred. (G) A CD spectrum of 145Stop showing a random-coil conformation. (H) Spontaneous LLPS of Y145Stop (100 $\mu \mathrm{M}, \mathrm{pH} 7.5,37^{\circ} \mathrm{C}$ ) from a homogeneous mixed phase into a demixed phase upon the addition of salt (350 mM NaCl). Confocal fluorescence images of homogeneous phase (I) and droplets (J) performed using Alexa488-labeled Y145Stop single-Cys mutant at residue 31. (K) Droplet fusions, surface-wetting, and dripping. (L) FRAP kinetics of multiple droplets (using 10\% Alexa488-labeled protein; $n=5$ ). The solid line represents the fitted curve (See Supporting Info for details). (M) Concentration estimation of the Y145Stop droplet (dense) phase and dispersed (light) phase using centrifugation assays. Data are represented as mean \pm SEM of independent measurements $(n=5)$. $(\mathrm{N})$ Comparison of phase separation ability of Y145Stop, full-length $\mathrm{PrP}$, and the C-terminal segment using turbidity and confocal microscopy. For 145Stop, the image shown in Figure 1J is included here for comparison.

using a bright, photostable, thiol-active fluorescent dye, AlexaFluor488 maleimide. Y145Stop mixed with fluorescently-labeled protein (1\%) was used for confocal imaging of droplets (Figure 1I,J). These fluorescent droplets undergo fusion, surface-wetting, dripping, and exhibit fast fluorescence recovery after photobleaching (FRAP) highlighting their liquidlike nature (Figure 1K,L). The concentration of protein inside droplets was estimated to be $2 \mathrm{mM}$, which is $\sim 25$ times denser compared to the dispersed phase $(\sim 80 \mu \mathrm{M})$ that corresponds to the critical saturation concentration, $\mathrm{C}_{\text {sat }}$ (Figure 1M). Fluorescence anisotropy measurements revealed no significant hindrance in the rotational relaxation of the polypeptide chain upon LLPS indicating a mobile interior within the droplets (data not shown). To mimic the cellular environment, we also studied phase separation of Y145Stop in the presence a of molecular crowding agent such as polyethylene glycol (PEG). In the presence of 10\% PEG, Y145Stop undergoes LLPS at a much lower protein concentration and at a physiological salt concentration $(150 \mathrm{mM})$ (Figure S1A).

Taken together, these results demonstrate that the N-terminal IDR of PrP, Y145Stop, under the near-physiological condition, undergoes condensation into liquid-like droplets with a protein-rich, dense, yet highly mobile, environment within the droplets. The net charge of this domain is +10.4 at $\mathrm{pH} 7.5$, and therefore, intermolecular repulsions between highly charged polypeptide chains prevent phase separation at low ionic strength. At higher salt concentrations, due to the effective charge-screening, polypeptide chains can commingle via 
weak, noncovalent, inter-chain interactions facilitating LLPS of this N-terminal IDR. Next, we asked what is the role of the C-terminal globular domain in modulating the prion phase behavior. PrP comprising both $\mathrm{N}$ - and C-terminal domains undergoes phase separation. ${ }^{57,58}$ However, under our condition, the phase separation propensity of the full-length PrP was much lower than the N-terminal IDR, Y145Stop (Figure 1N). This observation is consistent with the predictions (Figure 1E,F). We then created an N-terminally truncated form of the protein, PrP (112-231) which contains the entire C-terminal globular domain and a much shorter N-terminal tail comprising one of the two LCRs. As expected, this construct is wellfolded into a helical structure, as evident by CD (Figure S1B). PrP (112-231) did not phase separate even after prolonged incubation under our conditions in the presence or absence of salt. Our experimental data corroborate the results obtained from the prediction tools and suggest that the C-terminal globular domain alone is not prone to LLPS. In fact, the presence of this globular domain in full-length PrP diminishes the LLPS propensity. Together these observations indicated that the $\mathrm{N}$-terminal IDR is the principal driver of PrP phase separation, whereas, the C-terminal domain acts as an LLPS-inhibition domain indicating its chaperonelike role. Next, we set out to elucidate the key molecular determinants of phase separation of Y145Stop.

\section{Molecular drivers of phase separation of PrP Y145Stop}

Electrostatic screening. As stated above, Y145Stop carries a high net positive charge $(+10.4)$ at $\mathrm{pH}$ 7.5. Interchain repulsions prohibit intermolecular interactions that are essential for LLPS. In order to screen the high positive charge and to allow an effective interchain association, a threshold salt concentration is required above which it should phase separate via homotypic multivalent interactions. To unveil the role of electrostatic screening, we constructed the phase diagram as a function of salt concentration. Y145Stop phase separates above a critical threshold salt concentration (> $300 \mathrm{mM}, \mathrm{pH}$ 7.5) and remains phase-separated throughout the entire $\mathrm{NaCl}$ concentration range (Figures 2A,B, S2A). At a high salt concentration $(750 \mathrm{mM} \mathrm{NaCl})$, phase separation was observed at a much lower protein concentration (Figure 2B). In order to further support the role of electrostatic screening, we altered the charge-state of the protein by varying the solution $\mathrm{pH}$ at a fixed salt concentration.

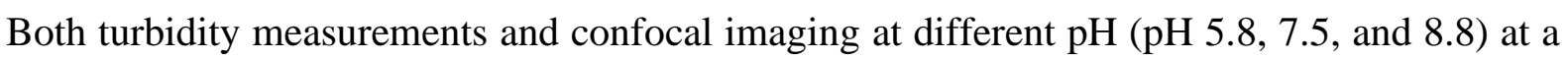
fixed ionic strength showed an enhanced phase separation with an increase in the $\mathrm{pH}$ (Figure $\mathrm{S} 2 \mathrm{~B}, \mathrm{C})$. The protein at a mildly acidic $\mathrm{pH}(\mathrm{pH} 5.8)$ carries a higher net positive charge ( 
+15 ), and therefore, requires a greater screening effect for phase separation compared to the protein at a mildly alkaline $\mathrm{pH}(\mathrm{pH} 8.8)$ containing a lower net positive charge $(\sim+8.5)$. These observations indicated that electrostatic screening is critical for phase separation of Y145Stop. An increase in the salt concentration decreases the Debye length allowing the screening of electrostatic repulsions between the positively charged residues resulting in the phase transition. ${ }^{59}$ Therefore, phase separation of Y145Stop is akin to 'salting out' like other phase separating proteins (TDP-43, hnRNPA1, and elastins) and is in contrast to the reentrant phase behavior in which LLPS is observed both at low salt and at high salt regime but not at intermediate salt concentrations. ${ }^{60-62}$ Since at high salt concentrations, the electrostatic interactions are negligible but the hydrophobic effect can be predominant, ${ }^{63,64}$ we next set out to characterize the role of hydrophobic interactions in driving phase separation of Y145Stop.
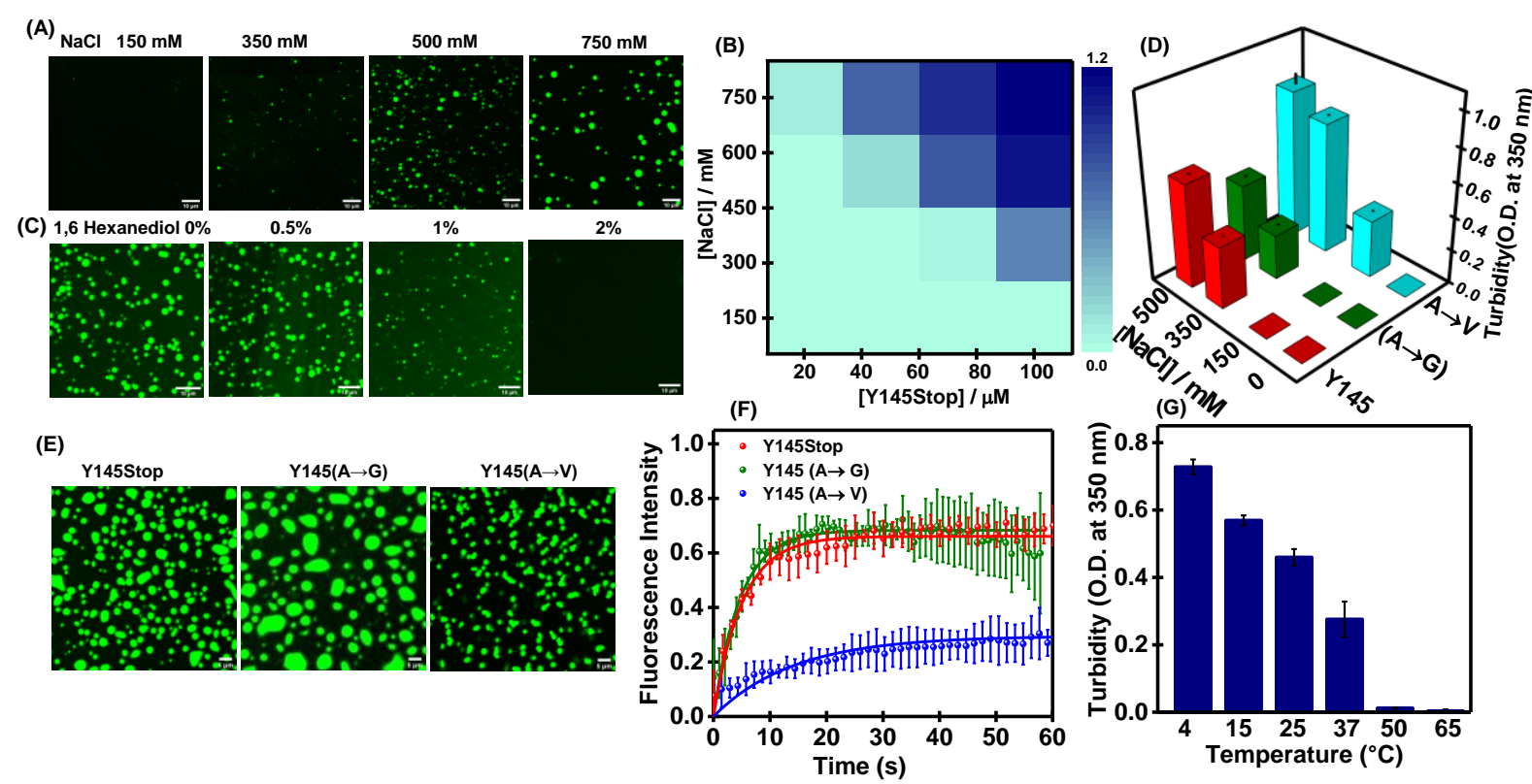

Figure 2. Molecular determinants of phase separation. (A) Confocal images of fluorescently labeled Y145Stop $\left(100 \mu \mathrm{M}, \mathrm{pH} 7.5,37^{\circ} \mathrm{C}\right)$ as a function increasing $\mathrm{NaCl}$ concentrations. (B) A phase diagram at different protein and salt concentrations constructed from mean turbidity values. (C) Confocal images of droplets in the presence of increasing amounts of 1,6 hexane diol (100 $\mu \mathrm{M}$ Y145Stop, $350 \mathrm{mM} \mathrm{NaCl}$, pH 7.5, $\left.37{ }^{\circ} \mathrm{C}\right)$. (D) Turbidity plot for Y145Stop, and variants $A \rightarrow G$ and $A \rightarrow V$ in presence of different concentrations of salt. (E) Confocal images of Y145Stop and variants $\mathrm{A} \rightarrow \mathrm{G}$ and $\mathrm{A} \rightarrow \mathrm{V}(100 \mu \mathrm{M}$ protein, $350 \mathrm{mM} \mathrm{NaCl}, \mathrm{pH} 7.5$, $37^{\circ} \mathrm{C}$ ) (F) FRAP kinetics of multiple fluorescently labeled droplets of Y145Stop and variants $\mathrm{A} \rightarrow \mathrm{G}$ and $\mathrm{Y} 145 \mathrm{~A} \rightarrow \mathrm{V} \quad(\mathrm{n}=5)$. (G) Phase separation of Y145Stop as a function of temperature. 
Hydrophobic interactions. In order to characterize the role of hydrophobic interactions in promoting phase separation, we first used 1,6-hexanediol, an aliphatic alcohol, that is known to disrupt weak hydrophobic interactions. Phase separation was inhibited by $2 \%(\mathrm{w} / \mathrm{v}) 1,6-$ hexanediol indicating an important role of hydrophobic interactions in LLPS of Y145Stop (Figure 2C, S3A). We also used ANS (8-anilino-1-naphthalenesulfonic acid) that is a wellknown hydrophobic reporter. We observed a blue-shift and an increase in the ANS fluorescence intensity upon LLPS of Y145Stop indicating the presence of local hydrophobic patches within the droplets (Figure S3B). A closer look at the amino acid sequence of Y145Stop revealed the presence of a hydrophobic stretch in the second LCR (residues 112121) that is rich in alanine and valine (Figure 1B). We further evaluated the role of hydrophobicity by performing site-directed mutagenesis at the hydrophobic stretch. We mutated residues by substituting three alanines to valines to increase the hydrophobicity and alanines to glycines to decrease the hydrophobicity. These mutations did not alter the structural attributes of Y145Stop as verified using CD measurements (Figure S3C). Glycine is polar and is a well-known spacer that does not appear to alter the saturation concentration. ${ }^{65}$ As expected, the substitution of alanines to glycines (Y145StopA $\rightarrow \mathrm{G}$ ) afforded droplets with a bit enhanced fluidity and behaved similarly to the wild-type Y145Stop (Figure 2D,E). However, the mutations of alanines to valines (Y145StopA $\rightarrow \mathrm{V}$ ) considerably enhanced the LLPS propensity and resulted in more solid-like and aggregationprone droplets as revealed by irregular shape in confocal microscopy associated with much lower FRAP recovery (Figure 2F). Thus, the mutations within the hydrophobic domain alter the phase behavior and simultaneously modulate the material properties of the assemblies. Together, these results indicate the role of hydrophobicity in driving the phase separation of Y145Stop. If the hydrophobic effect is a primary molecular driver of the phase transition, we would expect to observe higher phase separation propensity at higher temperatures due to the increase in the entropic contributions. Therefore, our next goal was to examine the thermoresponsive phase behavior of Y145Stop.

Thermo-responsive phase behavior. The interplay of molecular drivers of phase separation is critically dependent on the amino acid composition. ${ }^{20,65-68}$ Based on the amino acid composition, IDPs exhibit the upper critical solution transition (UCST) and the lower critical solution temperature (LCST) ${ }^{67}$ Polar LCRs interspersed by aromatic and charged residues display the UCST behavior, whereas, hydrophobic/aromatic-rich sequences follow the LCST behavior since the hydrophobic effect being largely entropic is more pronounced at a higher 
temperature. In order to characterize the temperature-dependent phase transition of Y145Stop, we performed the turbidity assay as a function of temperature ranging from $4{ }^{\circ} \mathrm{C}$ to $65{ }^{\circ} \mathrm{C}$. Interestingly, we observed an increase in the phase separation at lower temperatures indicating a UCST behavior (Figure 2G). These droplets exhibited temperature-dependent reversibility in the phase transition (Figure S4A). An LCST behavior is expected for LLPS that is primarily driven by hydrophobic interactions. The amino acid sequence of Y145Stop contains polar LCRs enriched in glycine, serine, glutamine, and asparagine, along with aromatic amino acids (tryptophan, tyrosine, and phenylalanine) and positively charged residues (lysine and arginine). The anomalous thermo-responsive characteristics plausibly point towards the dual behavior of aromatic amino acids central to both LCST and UCST transitions. These aromatic residues can participate in intermolecular $\pi-\pi$ stacking and cation$\pi$ interactions to promote LLPS. ${ }^{65}$ Our phase separation assays using free arginine showed that LLPS of Y145Stop is abolished at higher concentrations of arginine presumably due to strong interactions of free arginine with the aromatic sidechains that can potentially compete with intermolecular cation- $\pi$ interactions (Figure S4B,C). These results indicate the participation of aromatic residues in promoting phase separation of Y145Stop. Taken together, this set of studies highlight the presence of promiscuous interactions within the condensed environment of liquid-like droplets. The electrostatic screening allows the key noncovalent forces to promote transient, multivalent, interchain interactions between highly flexible conformers driving the liquid phase condensation of Y145Stop. Our next goal was to discern the key structural characteristics of polypeptide chains within the liquid droplets.

\section{Vibrational Raman spectroscopy captures the key conformational signatures}

In order to elucidate the protein conformational states, we employed vibrational Raman spectroscopy that provides a wealth of molecular information about the polypeptide backbones and sidechains. ${ }^{69,70}$ Additionally, a laser Raman assembly equipped with a microscope allows us to focus the laser beam into the protein-rich, dense, condensed phase of individual droplets to capture Raman scattering bands associated with a multitude of molecular vibrational modes from protein-rich single droplets. The Raman spectra of dispersed and phase-separated states of Y145Stop clearly showed characteristic bands corresponding to backbone amide I, amide III, Trp, Tyr, Phe, and other vibrational modes (Figure 3A). Amide I (1630-1700 $\mathrm{cm}^{-1}$ ) originates primarily due to the $\mathrm{C}=\mathrm{O}$ stretching of the polypeptide backbone, whereas, amide III (1230-1300 $\left.\mathrm{cm}^{-1}\right)$ corresponds to a combination of 
$\mathrm{N}-\mathrm{H}$ bending and C-N stretching modes. ${ }^{69,70}$ These amide vibrational bands are often used to identify the secondary structural elements in proteins. Broad amide I bands for both dispersed and demixed phases exhibited highly disordered conformations. A closer inspection of amide I revealed an increase in the FWHM (full-width at half maximum) from the dispersed phase $\left(\sim 55 \mathrm{~cm}^{-1}\right)$ to the droplet phase $\left(\sim 63 \mathrm{~cm}^{-1}\right)$ indicating a higher conformational heterogeneity in the condensed phase (Figure 3B). Next, we determined the intensity ratio of the tyrosine Fermi doublet $\left(\mathrm{I}_{850} / \mathrm{I}_{830}\right)$ that serves as an indicator of the hydrogen bonding strength between the phenolic hydroxyl group of Tyr and the neighboring water molecules in the vicinity. This ratio exhibited a small decrease from $\sim 2.75$ to $\sim 2.67$ upon phase separation indicating a small but measurable drop in the solvent accessibility in the liquid-like condensed phase. The intensity ratio for the tryptophan Fermi doublet $\left(\mathrm{I}_{1360} / \mathrm{I}_{1340}\right)$ (hydrophobicity) and a band at $880 \mathrm{~cm}^{-1}$ (hydrogen bonding strength between water and $\mathrm{N}-\mathrm{H}$ of the indole ring) did not exhibit any measurable changes upon the liquid phase condensation. We were able to recapitulate all these vibrational signatures in our single-droplet Raman studies. Taken together, these results demonstrate that Y145Stop retains its intrinsic disorder with a slight increase in the structural heterogeneity within the demixed liquid droplets.

(A)

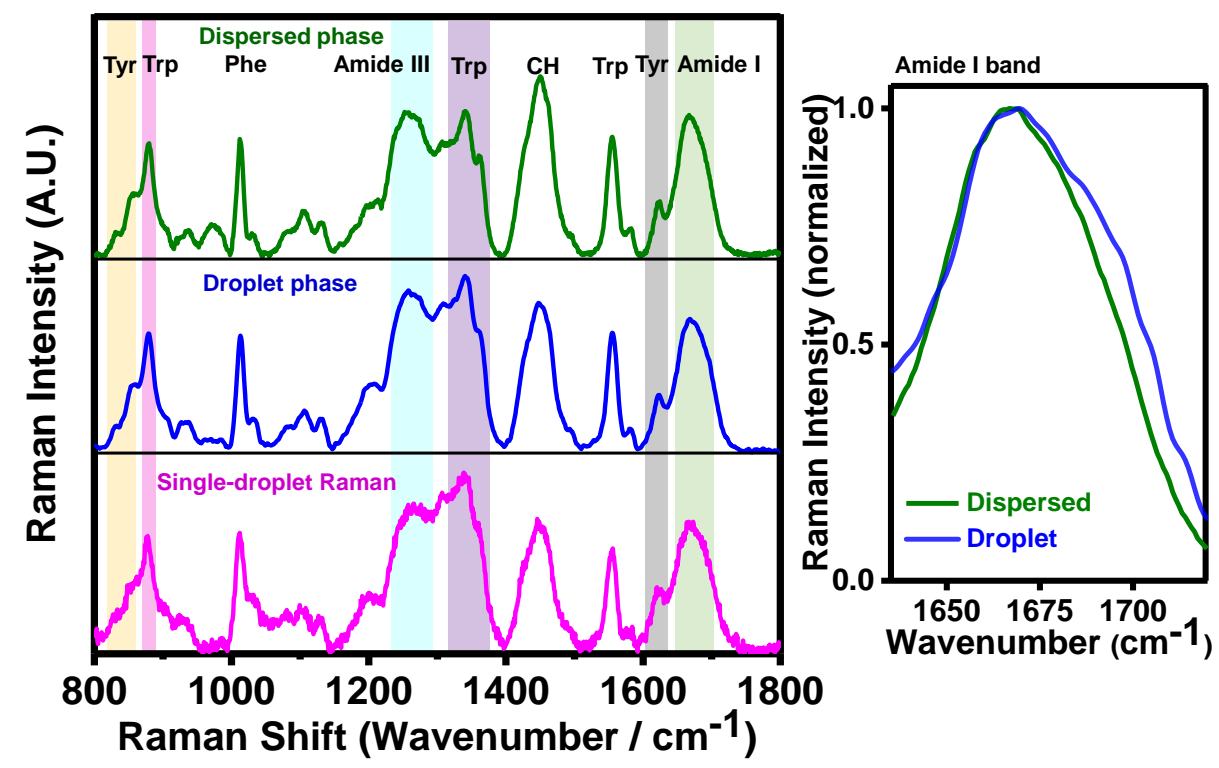

Figure 3. Vibrational Raman spectroscopy of Y145Stop. (A) Bulk Raman spectra of dispersed (olive) and droplet phases (blue) and single-droplet Raman (magenta). Prominent vibrational signatures of amide backbones and sidechains are shaded and labeled. (B) The amide I regions are shown for dispersed and condensed phases. 


\section{RNA regulates the phase behavior of Y145Stop}

The N-terminal IDR, Y145Stop, is broadly classified under the umbrella of RNA-binding proteins due to the presence of two highly conserved polybasic regions containing lysine clusters that have charge complementarity to nucleic acids. ${ }^{71,72} \mathrm{We}$ next examined the role of RNA in Y145Stop phase separation. The addition of crude t-RNA as low as 100 ng resulted in phase separation as confirmed using turbidity assays and confocal microscopy. The presence of RNA drastically lowered the saturation concentration of the protein to as low as 5 $\mu \mathrm{M}$ in the presence of $150 \mathrm{mM} \mathrm{NaCl}$ (Figure 4A,B). The droplets were initially spherical and rapidly transformed into a gel-like state comprising non-spherical condensates. We next performed FRAP experiments to investigate the protein diffusivity within these RNA-protein condensates. We observed much lower and incomplete recovery of these RNA-induced droplets $(\sim 20 \%)$ as compared to salt-induced droplets $(\sim 70 \%)$ indicating a more hardened material property of RNA-Y145Stop condensates (Figure 4C). In order to rule out the possibility of specificity of RNA in the observed phase behavior of Y145Stop, we also performed phase separation assays in the presence of other RNAs such as poly-U RNA and yeast total RNA. Our results showed a similar ability of these RNAs in modulating the phase behavior of Y145Stop (Figure S5). Next, to establish if the RNA-induced LLPS resulted in a more viscous, gel-like interior, we recovered apparent diffusion coefficients of the protein within the droplets (See Supporting Info).$^{34}$ In the absence of RNA, the FRAP recovery was on a faster timescale with a $\mathrm{t}_{1 / 2}$ of $3.48 \mathrm{~s}$ that yielded an approximated diffusion coefficient of $0.027 \mu \mathrm{m}^{2} \mathrm{~s}^{-1}$. The recovery kinetics in the presence of RNA was much slower $\left(\mathrm{t}_{1 / 2}=6.96 \mathrm{~s}\right)$ indicating a considerable drop in the apparent diffusion coefficient $\left(0.013 \mu \mathrm{m}^{2} \mathrm{~s}^{-1}\right)$. Therefore, the RNA-induced droplets are associated with lower protein diffusivity and enhanced internal viscosity. Next, we studied the effect of the RNA concentration on the phase separation of Y145Stop. We found that the phase separation ability of the protein is highly enhanced at a low RNA concentration, whereas, at higher concentrations of RNA, no phase separation was observed (Figure 4D,E). Protein solutions with high RNA concentrations remained dispersed and perfectly mixed even upon longer incubation. The interaction of poly-anions such as RNAs with the positively charged N-terminal domain possibly results in the charge inversion that prevents phase separation. Such an RNA stoichiometry-dependent promotion and inhibition of phase transition has been previously observed for FUS, TDP-43, LAF-1, and so forth. ${ }^{73-78}$ Together these results suggest that RNA buffers the phase behavior of Y145Stop and tune the material property of the condensates. 
(A)

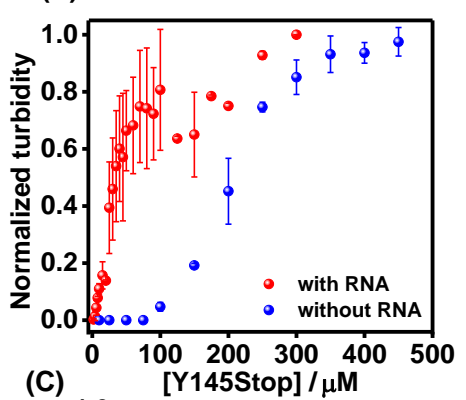

(C)

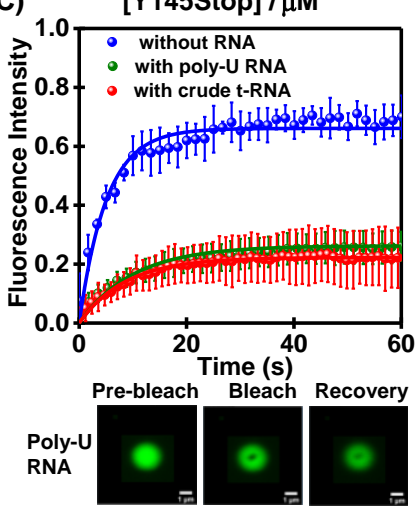

(B)

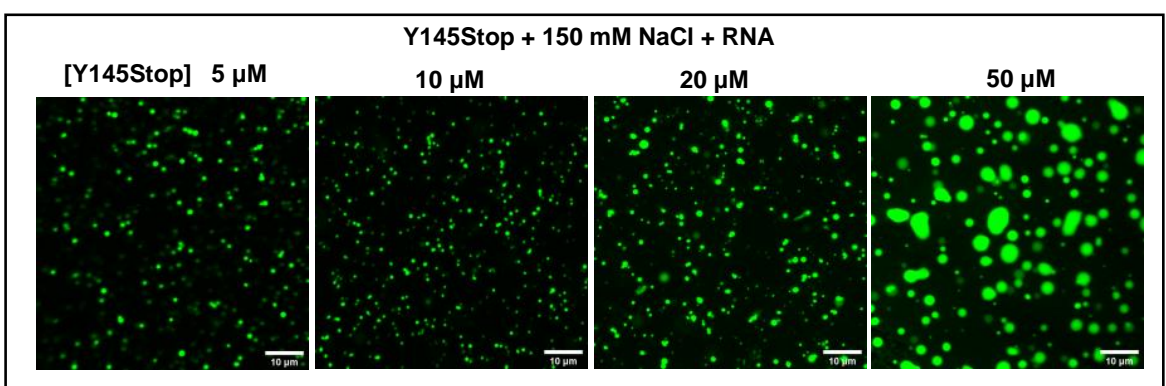

(D)

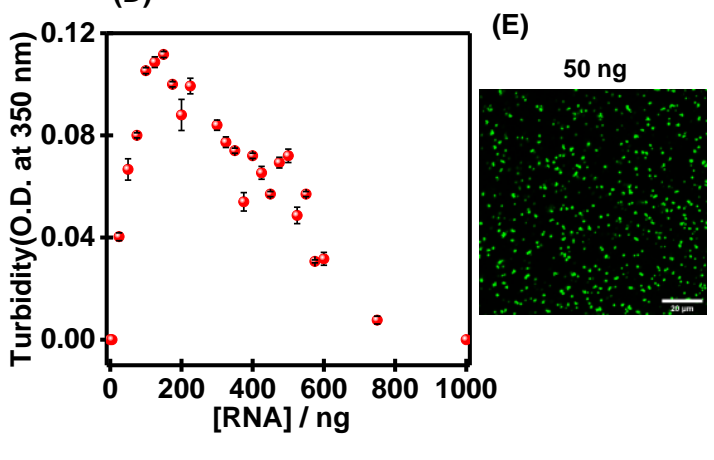

$100 \mathrm{ng}$

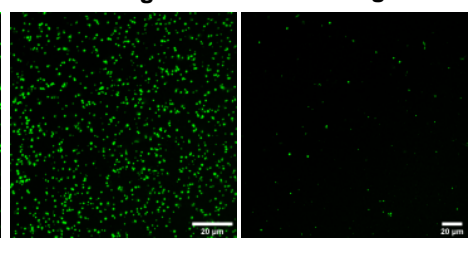

Figure 4. Modulation of Y145Stop phase transitions by RNA. RNA lowers the Y145Stop concentration required for phase separation from $\sim 80 \mu \mathrm{M}$ to $5 \mu \mathrm{M}$ of protein at $150 \mathrm{mM}$ $\mathrm{NaCl}$ and $\mathrm{pH} 7.5$ as observed by the turbidity assay (A) and confocal microscopy (B). (C) FRAP kinetics of multiple Y145Stop droplets (10\% Alexa488 labeled Y145Stop, $n=5)$ in the absence and the presence of RNA (crude t-RNA and poly-U RNA). The solid lines represent the fitted curves (see SI). The fluorescence images of droplets during FRAP measurements are also shown. (D \& E) Low RNA/protein ratios promote phase separation and high ratio inhibit phase separation at a fixed $(10 \mu \mathrm{M})$ Y145Stop concentration as observed by the RNAconcentration-dependent turbidity assays and confocal microscopy (poly-U RNA).

\section{Phase separation of Y145Stop promotes the formation of amyloid-like aggregates}

In order to monitor the maturation of Y145Stop condensates, we monitored the aging morphology of liquid droplets under a confocal microscope (Figure 5A). The liquid-like characteristics of these droplets were retained till $\sim 5 \mathrm{~h}$ after which these droplets irreversibly transformed into gel-like and/or solid-like fibrous morphologies. We observed 'sea urchin'like structures that were previously observed for FUS aggregates formed via LLPS. ${ }^{79}$ These results indicate that the droplets might act as the centers for nucleation and growth of fibers (Figure 5A). The solid-like nature of these aggregates was also confirmed using FRAP which 
(A)

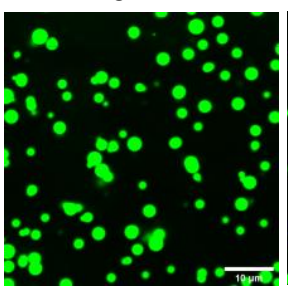

(B)
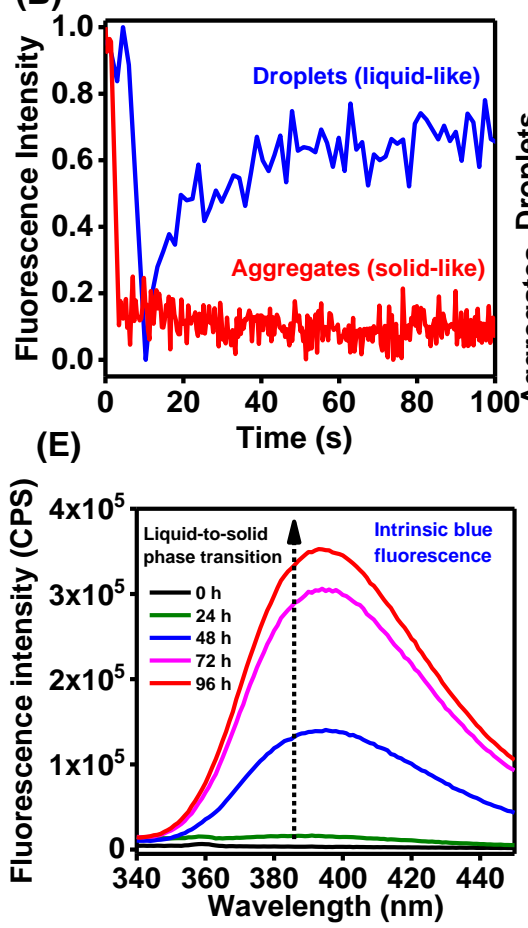

$4 \mathrm{~h}$

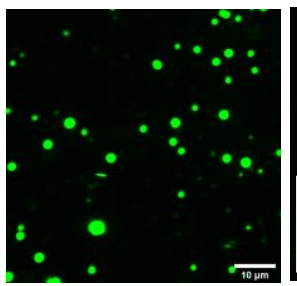

(C)

$12 \mathrm{~h}$

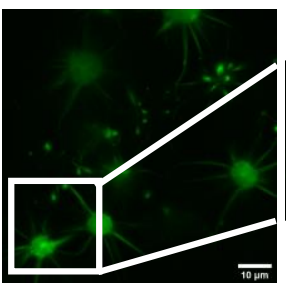

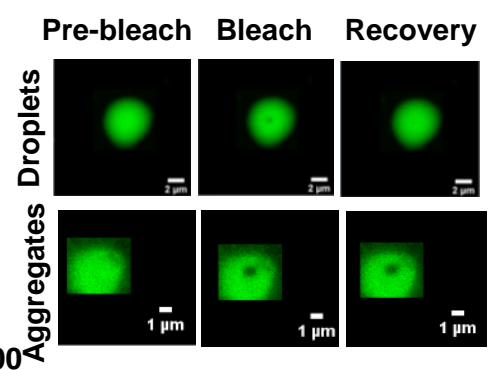

(F)

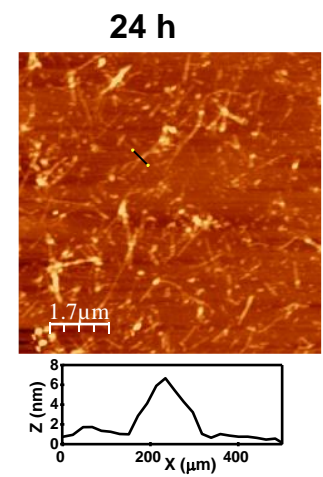

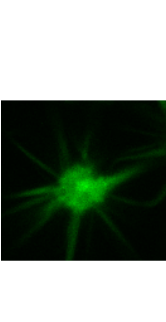

(D)
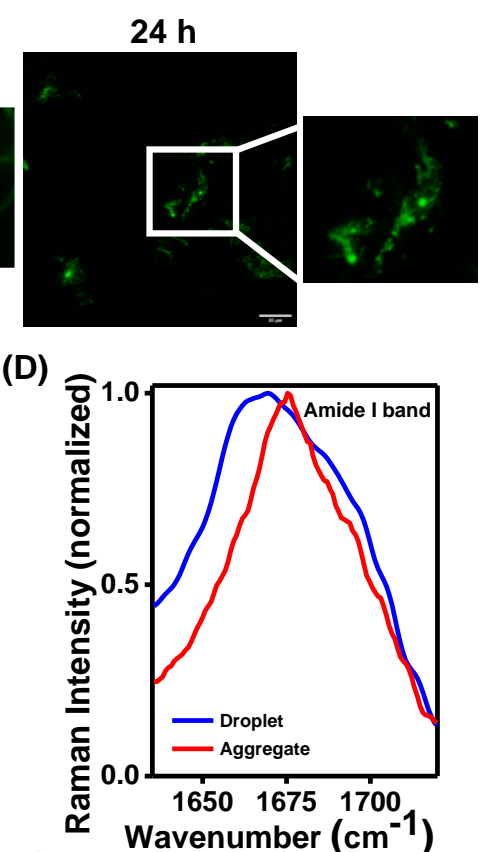

$48 \mathrm{~h}$

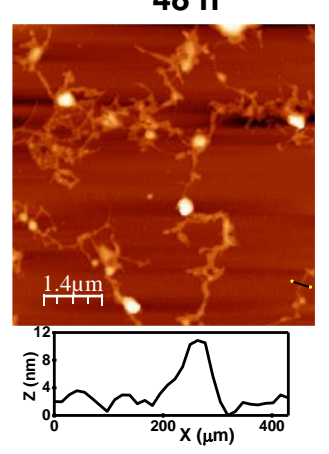
$60 \mathrm{~h}$

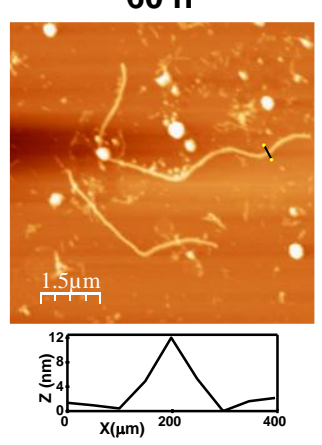

Figure 5. Liquid-to-solid phase transition of Y145Stop (A) Confocal images of fluorescently labeled Y145Stop showing morphological transitions as a function of time. (B) FRAP kinetics of solid-like aggregates showing no recovery (a representative FRAP kinetics of liquid-like state is also shown for comparison). (C) The fluorescence images during FRAP measurements. (D) Amide I vibrational Raman band showing a narrow peak at $1675 \mathrm{~cm}^{-1}$ indicating an amyloid-like cross- $\beta$ architecture. (E) Time-evolution of the intrinsic blue fluorescence during maturation. (F) Time-dependent AFM images of aggregates formed via LLPS showing the presence of typical amyloid fibrils with heights ranging from 8-12 nm.

showed no recovery (Figure 5B,C). These aggregates formed via the aging of liquid-like condensates are thioflavin-T (ThT) positive indicating a transition into amyloid-like aggregates (Figure S6A). Our vibrational Raman spectroscopic investigation showed a sharp amide I band at $1675 \mathrm{~cm}^{-1}$ that is a hallmark of hydrogen-bonded cross- $\beta$ architecture in 
amyloid fibrils (Figure 5D). ${ }^{70}$ The FWHM of amide I considerably narrowed down from liquid droplets $\left(\sim 63 \mathrm{~cm}^{-1}\right)$ to aggregates $\left(\sim 44 \mathrm{~cm}^{-1}\right)$ indicating a more ordered and less heterogeneous conformational state. The ratio of the tyrosine fermi doublet $\left(\mathrm{I}_{850} / \mathrm{I}_{830}\right)$ decreased considerably upon liquid-to-solid transition from droplets $(\sim 2.67)$ to aggregates $(\sim$ 2.16) suggesting a buried environment of Tyr residues with a lower propensity to form hydrogen bonds with water. Additionally, the polarity around Trp also exhibited a small decrease as evident by a slight blue shift of the band around $880 \mathrm{~cm}^{-1}$ indicating a lower hydrogen bonding ability of $\mathrm{N}-\mathrm{H}$ of the indole ring with water within the aggregates. Together these results suggested conformational sequestration of disordered polypeptide chains into a well-ordered amyloid architecture formed via liquid phase condensation and liquid-to-solid maturation. ${ }^{80}$ This LLPS-mediated liquid-to-solid transition is also characterized by the time-dependent evolution of intrinsic blue fluorescence (Figure 5E) that arises due to the intermolecular charge-transfer and electron delocalization via extensively hydrogen-bonded amide backbones within the phase-separated assemblies. ${ }^{81}$ Our atomic force microscopy (AFM) imaging revealed a fibrillar nanoscale morphology along with some amorphous aggregates (Figure 5F). Such amyloid fibrils with a diameter $~ 10 \mathrm{~nm}$ were also observed upon the phase transition and aging in the presence of RNA at a much lower protein concentration (Figure S6B). We also examined the aggregates formed via the aging of liquid droplets of the full-length PrP under the same condition. These PrP aggregates exhibited much lower ThT intensity compared to Y145Stop indicating the C-terminal folded domain lowers the aggregation propensity (Figure S6C). This observation hints at a protective, chaperone-like role of the C-terminal globular domain in the absence of which the protein undergoes an aberrant phase transition into amyloid-like aggregates. We next asked whether these amyloid aggregates formed via phase separation of Y145Stop can exhibit a selftemplating autocatalytic behavior that is a key characteristic of prion-like mechanisms.

\section{Aggregates formed via phase transition display a self-perpetuating autocatalytic behavior}

In order to probe the autocatalytic conversion, we first carried out a de novo aggregation reaction with Y145Stop that is known to proceed via a typical nucleation-dependent polymerization mechanism. ${ }^{47}$ The de novo aggregation kinetics exhibited a lag time $\sim 6 \mathrm{~h}$. Next, we carried out these kinetic experiments in the presence of increasing amounts of preformed seeds obtained from the salt-induced LLPS-mediated phase transition and aging for $24 \mathrm{~h}$. Upon addition of these seeds $(0.5 \%-10 \%)$, the lag phase shortened in a dose- 
dependent manner (Figure 6A). By using 10\% seeds in the reaction mixture, we were able to nearly bypass the lag phase ensuing a quasi-pseudo first-order kinetics. After establishing that the aggregates formed via phase separation are capable of recruiting and inducing a selfperpetuating conformational conversion, we asked which key species during liquid-to-solid maturation exhibit the most potent self-templating characteristics. In order to answer this question, we performed aggregation assays using the preformed seeds generated by the phase separation process followed by different aging times. Liquid droplets formed immediately upon LLPS exhibited much weaker seeding ability compared to the aged samples that were prepared by maturation into $\beta$-rich aggregates via liquid-to-solid transitions (Figure 6B). This observation suggests that amyloid conformers formed via the disorder-to-order phase transition are the key species in the autocatalytic conversion. The aggregation reaction seeded with aged solid-like species yielded typical amyloid fibrils reminiscent of nanoscale morphologies identical to fibrils formed via an unseeded de novo aggregation process (Figure 6C). Together these findings reveal that aberrant phase transitions of Y145Stop can produce self-replicating amyloid species recapitulating the bona fide prion-like behavior.
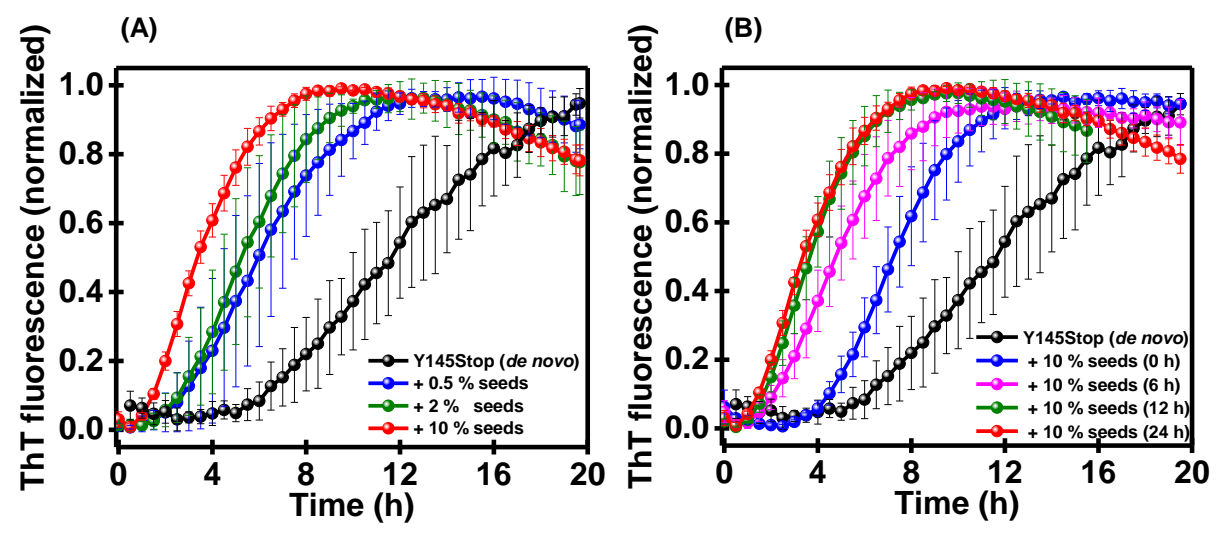

(C)

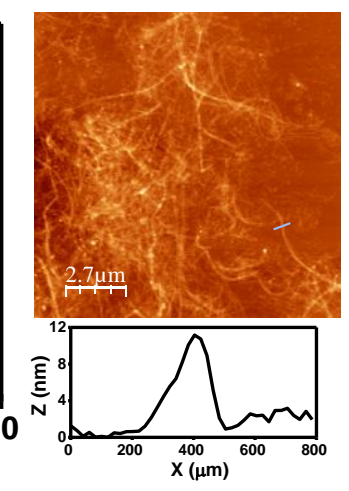

Figure 6. Seeding behavior of aggregates formed via LLPS. (A) The shortening of lag phase of de novo aggregation of Y145Stop $(100 \mu \mathrm{M})$ by the addition of increasing amounts of amyloid seeds obtained from phase separation of Y145Stop. (B) The seeding efficiency monitored as a function of aging of phase separated Y145Stop indicating the most effective seeding after phase maturation. (C) The AFM image of Y145Stop fibrils with the height profiles obtained after seeded assembly. 


\section{Discussion}

In this work, we showed that the intrinsically disordered PrP-145Stop containing oligopeptide repeats and LCRs undergoes an instantaneous phase separation at near-neutral $\mathrm{pH}$ concordant with the bioinformatic predictions. These phase-separated condensates formed immediately after inducing LLPS exhibit liquid-like characteristics having a highly mobile internal organization. The electrostatic screening of this highly positively charged N-terminal fragment at a higher salt concentration allowed the polypeptide chains to interact via weak and transient intermolecular contacts driving a reversible, thermo-responsive phase transition. The hydrophobic effect along with other noncovalent interactions serve as the physical crosslinks essential for phase transitions. Single-droplet Raman measurements revealed that the conformational disorder and structural heterogeneity are retained within the liquid-like droplets. Phase separation is further promoted by the addition of a molecular crowding agent at much lower protein and salt concentrations mimicking the cytosolic milieu. Upon aging, the liquid-like droplets gradually transform into irreversible gel-like and solid-like ordered fibrillar aggregates that exhibit amyloid-like characteristics. These amyloids formed via phase separation of Y145Stop display the self-templating autocatalytic behavior that is a key characteristic of the prion-like propagation mechanism. Full-length PrP containing both Nterminal IDR and C-terminal globular domain undergoes phase separation as observed before, ${ }^{57,82,83}$ albeit with a much lower propensity compared to Y145Stop under the same condition. This observation also corroborates the bioinformatic prediction. Additionally, the propensity for the liquid-to-solid transition into amyloid-like form is also much lower for the full-length protein than the N-terminal fragment. Taken together, our results showcase the crucial role of the Y145Stop variant comprising the N-terminal IDR in driving phase separation and aberrant phase transitions.

Our findings reveal that RNA critically modulates the phase behavior of Y145Stop that contains putative RNA binding sites. Low RNA/protein ratios promote phase separation at a much lower Y145Stop concentration $(5 \mu \mathrm{M})$ under the near-physiological condition $(\mathrm{pH}$ $7.5,150 \mathrm{mM} \mathrm{NaCl}$ ). RNA being a polyanion has a more pronounced influence in electrostatic neutralization of highly positively charged IDR than screening by salt ions in the absence of RNA. Therefore, RNA binding allows the chains to interact via a multitude of noncovalent interactions resulting in a complex coacervation. At low RNA/protein ratios, the RNAinduced droplets are more gel-like and rapidly transform into solid-like aggregates devoid of internal fluidity. In contrast, at higher RNA/protein ratios, the phase separation ability of 
Y145Stop was abolished. These observations suggested the stoichiometry of RNA and protein is critical in the RNA-dependent phase transition of Y145Stop. Our findings also highlight a broader role of RNA in aberrant phase transition and amyloid formation of Y145Stop that is associated with the GSS-like phenotype. The truncated Y145Stop variant is normally degraded by the proteasomal machinery or acted upon by the molecular chaperones. However, an impairment in the protein quality control allows this variant to accumulate in the endoplasmic reticulum, Golgi, and nucleus. Y145Stop overexpressed in cells treated with proteasome inhibitors has been shown to accumulate in the nucleus. ${ }^{51,84}$ Its presence in the nucleus is analogous to the aggregates found in diseases with polyglutamine repeats expansion. The presence of Y145Stop aggregates in the nucleus is intriguing as it has been ascribed to a cryptic nuclear localization signal which is otherwise masked in the full-length PrP. ${ }^{51}$

In summary, our study illuminates an intriguing interplay of molecular determinants that promote and regulate the phase transition and maturation into ordered self-templating amyloids from a pathological truncation variant of the prion protein. We would like to note that the putative functions of PrP such as copper homeostasis in the brain involve the binding of $\mathrm{Cu}^{2+}$ with the oligopeptide repeats located at the N-terminal IDR. ${ }^{85,86}$ Additionally, other proposed functions involving the maintenance of synaptic plasticity, suppressing apoptosis, resistance to oxidative stress are also located at the N-terminal domain. ${ }^{85,86}$ Even though the prion functions are mainly localized at the $\mathrm{N}$-terminal segment, the $\mathrm{C}$-terminal folded domain remains highly conserved during the vertebrate evolution. We, therefore, posit that the Cterminal globular domain can potentially have a protective chaperone-like activity to maintain the solubility of the functional $\mathrm{N}$-terminal domain preventing its aberrant phase transitions. Additionally, our results underscore the crucial role of phase-separated droplets as the reaction crucibles for efficient nucleation and effective sequestration into selfperpetuating amyloid conformers. These critical molecular events can have broad implications in biological phase transitions associated with physiology and disease. 


\section{Acknowledgments}

We thank IISER Mohali, Department of Science and Technology (Nano-Mission grant to S.M.), Department of Biotechnology (fellowship to A.A.), Council of Scientific and Industrial Research (fellowship to S.K.R.), Ministry of Education, Govt. of India (Centre of Excellence grant to S.M.) for financial support, Prof. Witold Surewicz (Case Western Reserve University) for kind gift of the DNA plasmid of full-length PrP, Dr. Mahak Sharma (IISER Mohali) for providing access to confocal microscope, Ms. Pallavi Joshi for preliminary studies, Dr. Shravan K. Mishra (IISER Mohali) for providing yeast total RNA, and Dr. Mily Bhattacharya (Thapar Institute) and the members of the Mukhopadhyay lab for critically reading this manuscript.

\section{References}

1. Choi, J.-M.; Holehouse, A. S.; Pappu, R. V., Physical principles underlying the complex biology of intracellular phase transitions. Annu. Rev. Biophys. 2020, 49, 107-133.

2. Sabari, B. R.; Dall'Agnese, A.; Young, R. A., Biomolecular condensates in the nucleus. Trends Biochem. Sci. 2020, 45, 961-977.

3. Alberti, S.; Dormann, D., Liquid-liquid phase separation in disease. Annu. Rev. Genet. 2019, 53, 171-194.

4. Alberti, S.; Gladfelter, A.; Mittag, T., Considerations and challenges in studying liquid-liquid phase separation and biomolecular condensates. Cell 2019, 176 (3), 419434.

5. Gomes, E.; Shorter, J., The molecular language of membraneless organelles. J. Biol. Chem. 2019, 294 (18), 7115-7127.

6. Bracha, D.; Walls, M. T.; Brangwynne, C. P., Probing and engineering liquid-phase organelles. Nat. Biotechnol. 2019, 37, 1435-1445.

7. Bentley, E. P.; Frey, B. B.; Deniz, A. A., Physical Chemistry of Cellular Liquid-Phase Separation. Chem. Eur. J. 2019, 25 (22), 5600-5610.

8. Boeynaems, S.; Alberti, S.; Fawzi, N. L.; Mittag, T.; Polymenidou, M.; Rousseau, F.; Schymkowitz, J.; Shorter, J.; Wolozin, B.; Van Den Bosch, L., Protein phase separation: a new phase in cell biology. Trends Cell Biol. 2018, 28 (6), 420-435. 
9. Forman-Kay, J. D.; Kriwacki, R. W.; Seydoux, G., Phase separation in biology and disease. J. Mol. Biol. 2018, 430 (23), 4603-4606.

10. Banani, S. F.; Lee, H. O.; Hyman, A. A.; Rosen, M. K., Biomolecular condensates: organizers of cellular biochemistry. Nat. Rev. Mol. Cell Biol. 2017, 18 (5), 285-298.

11. Uversky, V. N., Intrinsically disordered proteins in overcrowded milieu: Membraneless organelles, phase separation, and intrinsic disorder. Curr. Opin. Struct. Biol. 2017, $44,18-30$.

12. Mitrea, D. M.; Kriwacki, R. W., Phase separation in biology; functional organization of a higher order. Cell Commun. Signal. 2016, 14 (1), 1.

13. Hardenberg, M.; Horvath, A.; Ambrus, V.; Fuxreiter, M.; Vendruscolo, M., Widespread occurrence of the droplet state of proteins in the human proteome. Proc. Natl. Acad. Sci. U. S. A. 2020, 117 (52), 33254-33262..

14. Hyman, A. A.; Weber, C. A.; Jülicher, F., Liquid-liquid phase separation in biology. Ann. Rev. Cell Dev. Biol. 2014, 30, 39-58.

15. Toretsky, J. A.; Wright, P. E., Assemblages: functional units formed by cellular phase separation. J. Cell Biol. 2014, 206 (5), 579-588.

16. Hyman, A. A.; Brangwynne, C. P., Beyond stereospecificity: liquids and mesoscale organization of cytoplasm. Dev. Cell 2011, 21 (1), 14-16.

17. Posey, A. E.; Holehouse, A. S.; Pappu, R. V., Phase separation of intrinsically disordered proteins. Methods Enzymol. 2018, 611, 1-30.

18. Ruff, K. M.; Roberts, S.; Chilkoti, A.; Pappu, R. V., Advances in understanding stimulus-responsive phase behavior of intrinsically disordered protein polymers. $J$. Mol. Biol. 2018, 430 (23), 4619-4635.

19. Dignon, G. L.; Best, R. B.; Mittal, J., Biomolecular Phase Separation: From Molecular Driving Forces to Macroscopic Properties. Annu. Rev. Phys. Chem. 2020, 71, 53-75.

20. Martin, E. W.; Holehouse, A. S.; Peran, I.; Farag, M.; Incicco, J. J.; Bremer, A.; Grace, C. R.; Soranno, A.; Pappu, R. V.; Mittag, T., Valence and patterning of aromatic residues determine the phase behavior of prion-like domains. Science $\mathbf{2 0 2 0}$, 367 (6478), 694-699.

21. Hondele, M.; Heinrich, S.; De Los Rios, P.; Weis, K., Membraneless organelles: phasing out of equilibrium. Emerg. Top. Life Sci. 2020, 4 (3), 343-354.

22. Ranganathan, S.; Shakhnovich, E. I., Dynamic metastable long-living droplets formed by sticker-spacer proteins. Elife 2020, 9, e56159. 
23. Martin, E. W.; Holehouse, A. S., Intrinsically disordered protein regions and phase separation: sequence determinants of assembly or lack thereof. Emerg. Top. Life Sci. 2020, 4, 307-329.

24. Lin, Y.-H.; Forman-Kay, J. D.; Chan, H. S., Theories for sequence-dependent phase behaviors of biomolecular condensates. Biochemistry 2018, 57 (17), 2499-2508.

25. Brangwynne, C. P.; Tompa, P.; Pappu, R. V., Polymer physics of intracellular phase transitions. Nat. Phys. 2015, 11 (11), 899-904.

26. Lemke, E. A., The multiple faces of disordered nucleoporins. J. Mol. Biol. 2016, 428 (10), 2011-2024.

27. Mukhopadhyay, S., The Dynamism of Intrinsically Disordered Proteins: BindingInduced Folding, Amyloid Formation, and Phase Separation. J. Phys. Chem. B 2020, $124,11541-11560$.

28. Wong, L. E.; Kim, T. H.; Muhandiram, D. R.; Forman-Kay, J. D.; Kay, L. E., NMR experiments for studies of dilute and condensed protein phases: Application to the phase-separating protein CAPRIN1. J. Am. Chem. Soc. 2020, 142 (5), 2471-2489.

29. Shen, Y.; Ruggeri, F. S.; Vigolo, D.; Kamada, A.; Qamar, S.; Levin, A.; Iserman, C.; Alberti, S.; St George-Hyslop, P.; Knowles, T., Biomolecular condensates undergo a generic shear-mediated liquid-to-solid transition. bioRxiv $\mathbf{2 0 2 0 .}$

30. Elbaum-Garfinkle, S.; Brangwynne, C. P., Liquids, fibers, and gels: the many phases of neurodegeneration. Dev. Cell 2015, 35 (5), 531-532.

31. Elbaum-Garfinkle, S., Matter over mind: Liquid phase separation and neurodegeneration. J. Biol. Chem. 2019, 294 (18), 7160-7168.

32. Aguzzi, A.; Altmeyer, M., Phase separation: linking cellular compartmentalization to disease. Trends Cell Biol. 2016, 26 (7), 547-558.

33. Xing, Y.; Nandakumar, A.; Kakinen, A.; Sun, Y.; Davis, T. P.; Ke, P. C.; Ding, F., Amyloid Aggregation under the Lens of Liquid-Liquid Phase Separation. J. Phys. Chem. Lett. 2020, 12, 368-378.

34. Ray, S.; Singh, N.; Kumar, R.; Patel, K.; Pandey, S.; Datta, D.; Mahato, J.; Panigrahi, R.; Navalkar, A.; Mehra, S.; Gadhe, L.; Chatterjee, D.; Sawner, A. S.; Maiti, S.; Bhatia, S.; Gerez, J, A.; Chowdhury, A.; Kumar, A.; Padinhateeri, R.; Riek, R.; Krishnamoorthry, G.; Maji, S. K., $\alpha$-Synuclein aggregation nucleates through liquidliquid phase separation. Nat. Chem. 2020, 12 (8), 705-716.

35. Alberti, S., The wisdom of crowds: regulating cell function through condensed states of living matter. J. Cell Sci. 2017, 130 (17), 2789-2796. 
36. Boeynaems, S.; Bogaert, E.; Kovacs, D.; Konijnenberg, A.; Timmerman, E.; Volkov, A.; Guharoy, M.; De Decker, M.; Jaspers, T.; Ryan, V. H.; Janke, A. M.; Baatsen, P.; Vercruysse, T.; Kolaitis, R. M.; Daelemans, D.; Taylor, J. P.; Kedersha, N.; Anderson, P.; Impens, F.; Sobott, F.; Schymkowitz, J.; Rousseau, F.; Fawzi, N. L.; Robberecht, W.; Van, D. P.; Tompa, P.; Van Den Bosch, L., Phase separation of C9orf72 dipeptide repeats perturbs stress granule dynamics. Mol. Cell 2017, 65 (6), 1044-1055.

37. Ambadipudi, S.; Biernat, J.; Riedel, D.; Mandelkow, E.; Zweckstetter, M., Liquidliquid phase separation of the microtubule-binding repeats of the Alzheimer-related protein Tau. Nat. Commun. 2017, 8 (1), 1-13.

38. Tsang, B.; Pritišanac, I.; Scherer, S. W.; Moses, A. M.; Forman-Kay, J. D., Phase Separation as a Missing Mechanism for Interpretation of Disease Mutations. Cell 2020, 183 (7), 1742-1756.

39. Bhopatkar, A. A.; Uversky, V. N.; Rangachari, V., Granulins modulate liquid-liquid phase separation and aggregation of the prion-like C-terminal domain of the neurodegeneration-associated protein TDP-43. J. Biol. Chem. 2020, 295 (8), 25062519.

40. Prusiner, S. B., Prions. Proc. Natl. Acad. Sci. U. S. A. 1998, 95 (23), 13363-13383.

41. Prusiner, S. B., Prion biology. Cold Spring Harbour Laboratory Press: 2017.

42. Scheckel, C.; Aguzzi, A., Prions, prionoids and protein misfolding disorders. Nat. Rev. Genet. 2018, 19 (7), 405-418.

43. Zahn, R.; Liu, A.; Lührs, T.; Riek, R.; von Schroetter, C.; García, F. L.; Billeter, M.; Calzolai, L.; Wider, G.; Wüthrich, K., NMR solution structure of the human prion protein. Proc. Natl. Acad. Sci. U. S. A. 2000, 97 (1), 145-150.

44. Castilla, J.; Saá, P.; Hetz, C.; Soto, C., In vitro generation of infectious scrapie prions. Cell 2005, 121 (2), 195-206.

45. Deleault, N. R.; Harris, B. T.; Rees, J. R.; Supattapone, S., Formation of native prions from minimal components in vitro. Proc. Natl. Acad. Sci. U. S. A. 2007, 104 (23), 9741-9746.

46. Wang, F.; Wang, X.; Yuan, C.-G.; Ma, J., Generating a prion with bacterially expressed recombinant prion protein. Science 2010, 327 (5969), 1132-1135.

47. Kundu, B.; Maiti, N. R.; Jones, E. M.; Surewicz, K. A.; Vanik, D. L.; Surewicz, W. K., Nucleation-dependent conformational conversion of the Y145Stop variant of 
human prion protein: structural clues for prion propagation. Proc. Natl. Acad. Sci. U. S. A. 2003, 100 (21), 12069-12074.

48. Helmus, J. J.; Surewicz, K.; Nadaud, P. S.; Surewicz, W. K.; Jaroniec, C. P., Molecular conformation and dynamics of the Y145Stop variant of human prion protein in amyloid fibrils. Proc. Natl. Acad. Sci. U. S. A. 2008, 105 (17), 6284-6289.

49. Zweckstetter, M., Conserved amyloid core structure of stop mutants of the human prion protein. Prion 2013, 7 (3), 193-197.

50. Ghetti, B.; Piccardo, P.; Spillantini, M. G.; Ichimiya, Y.; Porro, M.; Perini, F.; Kitamoto, T.; Tateishi, J.; Seiler, C.; Frangione, B., Vascular variant of prion protein cerebral amyloidosis with tau-positive neurofibrillary tangles: the phenotype of the stop codon 145 mutation in PRNP. Proc. Natl. Acad. Sci. U. S. A. 1996, 93 (2), 744748.

51. Zanusso, G.; Petersen, R. B.; Jin, T.; Jing, Y.; Kanoush, R.; Ferrari, S.; Gambetti, P.; Singh, N., Proteasomal degradation and N-terminal protease resistance of the codon 145 mutant prion protein. J. Biol. Chem. 1999, 274 (33), 23396-23404.

52. Xue, B.; Dunbrack, R. L.; Williams, R. W.; Dunker, A. K.; Uversky, V. N., PONDRFIT: a meta-predictor of intrinsically disordered amino acids. Biochim. Biophys. Acta, Proteins Proteomics 2010, 1804 (4), 996-1010.

53. Mészáros, B.; Erdős, G.; Dosztányi, Z., IUPred2A: context-dependent prediction of protein disorder as a function of redox state and protein binding. Nucleic Acids Res. 2018, 46 (W1), W329-W337.

54. Miskei, M.; Horvath, A.; Vendruscolo, M.; Fuxreiter, M., Sequence-based prediction of fuzzy protein interactions. J. Mol. Biol. 2020, 432 (7), 2289-2303.

55. Bolognesi, B.; Gotor, N. L.; Dhar, R.; Cirillo, D.; Baldrighi, M.; Tartaglia, G. G.; Lehner, B., A concentration-dependent liquid phase separation can cause toxicity upon increased protein expression. Cell Rep. 2016, 16 (1), 222-231.

56. Letunic, I.; Bork, P., 20 years of the SMART protein domain annotation resource. Nucleic Acids Res. 2018, 46, D493-D496.

57. Kostylev, M. A.; Tuttle, M. D.; Lee, S.; Klein, L. E.; Takahashi, H.; Cox, T. O.; Gunther, E. C.; Zilm, K. W.; Strittmatter, S. M., Liquid and hydrogel phases of PrPC linked to conformation shifts and triggered by alzheimer's amyloid- $\beta$ oligomers. Mol. Cell. 2018, 72 (3), 426-443.

58. Matos, C. O.; Passos, Y. M.; do Amaral, M. J.; Macedo, B.; Tempone, M. H.; Bezerra, O. C.; Moraes, M. O.; Almeida, M. S.; Weber, G.; Missailidis, S., 
Liquid-liquid phase separation and fibrillation of the prion protein modulated by a high-affinity DNA aptamer. FASEB J. 2020, 34 (1), 365-385.

59. Smith, A. M.; Lee, A. A.; Perkin, S., The electrostatic screening length in concentrated electrolytes increases with concentration. J. Phys. Chem. Lett. 2016, 7 (12), 2157-2163.

60. Babinchak, W. M.; Haider, R.; Dumm, B. K.; Sarkar, P.; Surewicz, K.; Choi, J.-K.; Surewicz, W. K., The role of liquid-liquid phase separation in aggregation of the TDP-43 low-complexity domain. J. Biol. Chem. 2019, 294 (16), 6306-6317.

61. Milkovic, N. M.; Thomasen, F. E.; Cuneo, M. J.; Grace, C. R.; Martin, E. W.; Nourse, A.; Lindorff-Larsen, K.; Mittag, T., Interplay of folded domains and the disordered low-complexity domain in mediating hnRNPA1 phase separation. bioRxiv $\mathbf{2 0 2 0}$.

62. Reichheld, S. E.; Muiznieks, L. D.; Keeley, F. W.; Sharpe, S., Direct observation of structure and dynamics during phase separation of an elastomeric protein. Proc. Natl. Acad. Sci. U. S. A. 2017, 114 (22), E4408-E4415.

63. Krainer, G.; Welsh, T. J.; Joseph, J. A.; Espinosa, J. R.; de Csillery, E.; Sridhar, A.; Toprakcioglu, Z.; Gudiskyte, G.; Czekalska, M. A.; Arter, W. E., Reentrant liquid condensate phase of proteins is stabilized by hydrophobic and non-ionic interactions. bioRxiv 2020.

64. Conicella, A. E.; Dignon, G. L.; Zerze, G. H.; Schmidt, H. B.; Alexandra, M.; Kim, Y. C.; Rohatgi, R.; Ayala, Y. M.; Mittal, J.; Fawzi, N. L., TDP-43 $\alpha$-helical structure tunes liquid-liquid phase separation and function. Proc. Natl. Acad. Sci. U. S. A. 2020, 117 (11), 5883-5894.

65. Wang, J.; Choi, J.-M.; Holehouse, A. S.; Lee, H. O.; Zhang, X.; Jahnel, M.; Maharana, S.; Lemaitre, R.; Pozniakovsky, A.; Drechsel, D.; Poser, I.; Pappu, R. V.; Alberti, S.; Hymann, A. A., A molecular grammar governing the driving forces for phase separation of prion-like RNA binding proteins. Cell 2018, 174 (3), 688-699.

66. Qamar, S.; Wang, G.; Randle, S. J.; Ruggeri, F. S.; Varela, J. A.; Lin, J. Q.; Phillips, E. C.; Miyashita, A.; Williams, D.; Ströhl, F.; Meadows, W.; Ferry, R.; Dardov, V. J.; Tartaglia, G. G.; Farrer, L. A.; Kaminski, C. F.; Holt, C. E.; Fraser, P. E.; SchmittiUlms, G.; Klenerman, D.; Knowles, T.; Vendruscolo, M.; St George-hyslop, P., FUS phase separation is modulated by a molecular chaperone and methylation of arginine cation- $\pi$ interactions. Cell 2018, 173 (3), 720-734.

67. Martin, E. W.; Mittag, T., Relationship of sequence and phase separation in protein low-complexity regions. Biochemistry 2018, 57 (17), 2478-2487. 
68. Quiroz, F. G.; Li, N. K.; Roberts, S.; Weber, P.; Dzuricky, M.; Weitzhandler, I.; Yingling, Y. G.; Chilkoti, A., Intrinsically disordered proteins access a range of hysteretic phase separation behaviors. Sci. Adv. 2019, 5 (10), eaax5177.

69. Tuma, R., Raman spectroscopy of proteins: from peptides to large assemblies. $J$. Raman Spectrosc. 2005, 36 (4), 307-319.

70. Bhattacharya, M.; Jain, N.; Dogra, P.; Samai, S.; Mukhopadhyay, S., Nanoscopic amyloid pores formed via stepwise protein assembly. J. Phys. Chem. Lett. 2013, 4 (3), 480-485.

71. Lathe, R.; Darlix, J.-L., Prion protein PrP nucleic acid binding and mobilization implicates retroelements as the replicative component of transmissible spongiform encephalopathy. Arch. Virol. 2020, 165, 535-556.

72. Gabus, C.; Derrington, E.; Leblanc, P.; Chnaiderman, J.; Dormont, D.; Swietnicki, W.; Morillas, M.; Surewicz, W. K.; Marc, D.; Nandi, P., The prion protein has RNA binding and chaperoning properties characteristic of nucleocapsid protein NCP7 of HIV-1. J. Biol. Chem. 2001, 276 (22), 19301-19309.

73. Hofweber, M.; Hutten, S.; Bourgeois, B.; Spreitzer, E.; Niedner-Boblenz, A.; Schifferer, M.; Ruepp, M.-D.; Simons, M.; Niessing, D.; Madl, T.; Dormann, D., Phase separation of FUS is suppressed by its nuclear import receptor and arginine methylation. Cell 2018, 173 (3), 706-719.

74. Banerjee, P. R.; Milin, A. N.; Moosa, M. M.; Onuchic, P. L.; Deniz, A. A., Reentrant phase transition drives dynamic substructure formation in ribonucleoprotein droplets. Angew. Chem., Int. Ed. 2017, 129 (38), 11512-11517.

75. Alshareedah, I.; Kaur, T.; Ngo, J.; Seppala, H.; Kounatse, L.-A. D.; Wang, W.; Moosa, M. M.; Banerjee, P. R., Interplay between Short-Range Attraction and LongRange Repulsion Controls Reentrant Liquid Condensation of RibonucleoproteinRNA Complexes. J. Am. Chem. Soc. 2019, 141 (37), 14593-14602.

76. Lin, Y.; Protter, D. S.; Rosen, M. K.; Parker, R., Formation and maturation of phaseseparated liquid droplets by RNA-binding proteins. Mol. Cell 2015, 60 (2), 208-219.

77. Elbaum-Garfinkle, S.; Kim, Y.; Szczepaniak, K.; Chen, C. C.-H.; Eckmann, C. R.; Myong, S.; Brangwynne, C. P., The disordered P granule protein LAF-1 drives phase separation into droplets with tunable viscosity and dynamics. Proc. Natl. Acad. Sci. U. S. A. 2015, 112 (23), 7189-7194.

78. Ukmar-Godec, T.; Hutten, S.; Grieshop, M. P.; Rezaei-Ghaleh, N.; Cima-Omori, M.S.; Biernat, J.; Mandelkow, E.; Söding, J.; Dormann, D.; Zweckstetter, M., 
Lysine/RNA-interactions drive and regulate biomolecular condensation. Nat. Commun. 2019, 10 (1), 1-15.

79. Patel, A.; Lee, H. O.; Jawerth, L.; Maharana, S.; Jahnel, M.; Hein, M. Y.; Stoynov, S.; Mahamid, J.; Saha, S.; Franzmann, T. M.; Pozniakovski, A.; Poser, I.; Maghelli, N.; Royer, L. A.; Weigert, M.; Myers, E. W.; Grill, S.; Drechsel, D.; Hyman, A. A.; Alberti, S., A liquid-to-solid phase transition of the ALS protein FUS accelerated by disease mutation. Cell 2015, 162 (5), 1066-1077.

80. Murthy, A. C.; Dignon, G. L.; Kan, Y.; Zerze, G. H.; Parekh, S. H.; Mittal, J.; Fawzi, N. L., Molecular interactions underlying liquid- liquid phase separation of the FUS low-complexity domain. Nat. Struct. Mol. Biol. 2019, 26 (7), 637-648.

81. Dogra, P.; Joshi, A.; Majumdar, A.; Mukhopadhyay, S., Intermolecular ChargeTransfer Modulates Liquid-Liquid Phase Separation and Liquid-to-Solid Maturation of an Intrinsically Disordered pH-Responsive Domain. J. Am. Chem. Soc. 2019, 141 (51), 20380-20389.

82. Tange, H.; Ishibashi, D.; Nakagaki, T.; Taguchi, Y.; Kamatari, Y. O.; Nishida, N.; Ozawa, H., Phase transition of prion protein initiates proteinase $\mathrm{K}$ resistant amyloid formation. bioRxiv $\mathbf{2 0 2 0 .}$

83. Huang, J.-J.; Li, X.-N.; Liu, W.-L.; Yuan, H.-Y.; Gao, Y.; Wang, K.; Tang, B.; Pang, D.-W.; Chen, J.; Liang, Y., Neutralizing mutations significantly inhibit amyloid formation by human prion protein and decrease its cytotoxicity. J. Mol. Biol. 2020, $432(4), 828-844$.

84. Lorenz, H.; Windl, O.; Kretzschmar, H. A., Cellular phenotyping of secretory and nuclear prion proteins associated with inherited prion diseases. J. Biol. Chem. 2002, 277 (10), 8508-8516.

85. Wulf, M.-A.; Senatore, A.; Aguzzi, A., The biological function of the cellular prion protein: an update. BMC Biol. 2017, 15 (1), 1-13.

86. Wu, B.; McDonald, A. J.; Markham, K.; Rich, C. B.; McHugh, K. P.; Tatzelt, J.; Colby, D. W.; Millhauser, G. L.; Harris, D. A., The N-terminus of the prion protein is a toxic effector regulated by the C-terminus. elife 2017, 6, e23473. 